\title{
Green Data: Estrategia comunicativa en los nuevos medios, una educación informal para combatir el calentamiento global
}

\author{
Héctor Iván Alzugaray Cedillo, Alan Borayín Ortiz Ramírez, Frida Karen González Martínez, \\ Karla Lizzet Guardado Espejel, Itzel Susana Pérez Cornejo ${ }^{1}$
}

\begin{abstract}
Resumen
Una estrategia comunicativa de educación informal, a través de productos educomunicativos busca incidir en la concientización de la sociedad, debido a la problemática ambiental que significa el fenómeno del calentamiento global, explicando el mismo y su relación con la conducta humana en diversos contextos. Por medio de cápsulas audiovisuales distribuidas en redes sociales y dirigidas, principalmente, a la generación Millennial, se logró alcanzar a más de 30 mil personas en más de 10 países.

Abstract

This thesis created communicative strategy of informal education, through the educommunicative products, to influence the awareness of society in the significance of the global warming phenomena and the environmental problems it implies. The project explains it and its relationship with human behavior in different contexts. It reached more than 30 thousand people in more than 10 countries through audiovisual capsules, distributed in social media and mainly aimed at the Millennial generation.
\end{abstract}

\section{Palabras Clave}

Educomunicación, Calentamiento Global, Millennials, Estrategia Comunicativa, Redes Sociales

Keywords

Educommunication, Global Warming, Millennials, Communication Strategy, Social Media

Segundo lugar del Concurso de Trabajos Recepcionales en Comunicación CONEICC 2018. Universidad Autónoma Metropolitana Xochimilco. Asesor responsable: Dr. Mauricio Andión Gamboa. Asesor interno: Mtro: Marco Diego Vargas Ugalde. Asesor externo: Dra. Rosario Casco Montoya.

${ }^{1}$ Autor para correspondencia: ispc13@gmail.com

\section{Problemática de investigación}

El calentamiento global es un problema a nivel mundial que en los últimos años ha cobrado una importancia significativa, pues su presencia ha perturbado la vida de todas las especies, tanto terrestres como acuáticas, entre las cuales también se encuentra el ser humano.

Por lo tanto, se debe entender dicho fenómeno a través de su vigencia, pertinencia y relevancia, siendo estos diversos niveles que dan pie al entendimiento de la problemática que abarca este trabajo de investigación.

\subsection{Vigencia}

En el 2018, el calentamiento global es ya un problema urgente, real, peligroso, y del que se debe tener conciencia en cuanto al nivel de consumo y lo que se realiza para que esto frene o continúe, pues se estima que los seres humanos consumen los recursos equivalentes a una Tierra y media, por lo que las Naciones Unidas calcula que, si el crecimiento demográfico y las tendencias de consumo siguen como hasta ahora, para el año 2030, se requerirán el equivalente a dos planetas como la Tierra para regenerar los recursos que se gastan (Rifkin, 2015). 
Es un hecho que el calentamiento global es un fenómeno existente que lleva sucediendo mucho tiempo y no se ha logrado detener. Sin embargo, hoy en día se vuelve un tema imperioso y es momento de que se preste la debida atención en la conducta humana respecto a la biosfera, los hábitos que tiene con ella y la cultura ambiental de la cual se carece en la actualidad.

\subsection{Pertinencia}

La problemática del calentamiento global conlleva una tarea ardua puesto que implica cambiar la mentalidad y los esquemas de acción de la sociedad. Ante esta situación, el proyecto Green Data se crea como una propuesta capaz de utilizar la comunicación multimedia como un vehículo idóneo para planear, elaborar y distribuir objetos comunicativos enfocados a la educación ambiental, mediante estrategias pedagógicas informales, con las cuales, busca no sólo educar en el ámbito ecológico, sino también, fomentar el desarrollo de una conciencia crítica, capaz de identificar, reflexionar y actuar en torno a los problemas ambientales más relevantes de la actualidad.

Dicho proyecto multimedia se ve enfocado en gran parte a los Millenials, quienes representan un porcentaje importante de la fuerza laboral presente y futura, siendo ellos la población que va de los 12 a los 29 años de edad, conformando el $31.4 \%$ de la población total en México, por lo que constituyen un grupo cuyo papel en el consumo de bienes y servicios, así como en el cuidado de la biosfera. Será determinante además de que una de las principales cualidades de este grupo de la población se encuentra su forma de relacionarse con la tecnología, pues poseen una predilección y adaptabilidad a los nuevos medios.

\subsection{Relevancia}

La educación ambiental es una de las principales preocupaciones que deben implantarse a fondo en la conciencia de todos los seres humanos, para así lograr un cambio antes de que las condiciones climatológicas impidan la vida en el planeta. De acuerdo con la Organización de las Naciones Unidas (ONU), los años 2015, 2016 y 2017 han sido los más calurosos de los que se tiene registro hasta ahora, lo que indica que el cambio climático es cada vez más evidente y va en aumento (Greenpeace, 2017).
La concientización, en este sentido, debe ser prioritaria, con programas y proyectos de esta índole que sean aplicados por los gobiernos mundiales, para que la acción ecológica y de preservación de la biosfera pueda ser mayor y el cambio se produzca de manera sustancial, llevando a un cambio en los valores de consumo, siempre en pro de la naturaleza y su conservación.

\section{Contextos de referencia}

La urgencia de un cambio que impone el calentamiento global se refiere a todos los ámbitos que en conjunto conforman el día a día de la humanidad, entendiéndose por estos lo social, lo político, lo económico, lo cultural y desde luego, lo ambiental. Además, sería posible impactar en dichos ámbitos si se genera una cadena de cambios que inicie desde cualquiera de estos aspectos, en los cuales la biosfera tiene un papel vital, por lo cual, es necesario entender la situación actual en cada uno de ellos y el por qué se ha propiciado la destrucción de la naturaleza, para posteriormente generar alternativas que den pie a un cambio real.

\subsection{Contexto ambiental}

Se le denomina calentamiento global al aumento gradual de la temperatura media en los océanos y en la atmósfera del planeta Tierra. Este incremento de temperatura se debe, entre otros factores, principalmente a la actividad humana, provocando que la cantidad de gases de efecto invernadero sea mayor, y esta se acumule en la superficie del planeta. Dichas actividades van desde la explotación de los recursos naturales hasta la contaminación del aire debido a la liberación de gases químicos que generan diversas industrias. Las consecuencias que genera el calentamiento global por medio del aumento de la temperatura no sólo significa más calor para los seres vivos, sino que también derrite los glaciares y otras masas de hielos en todo el planeta, que a su vez se traduciría en un riesgo importante en las reservas de agua dulce en el mundo, el crecimiento del nivel del mar, entre otras, tan sólo por mencionar uno de sus efectos.

Por lo anterior, es que los seres humanos deben fungir como agentes de cambio en el calentamiento global, para restaurar y frenar el daño al planeta Tierra. Pequeñas acciones no son de utilidad si se llevan a cabo sin una conciencia colectiva, por lo que la organización política, social y 
económica, debe girar en torno a la biosfera. Tal como lo menciona Naomi Klein: "Si de verdad nos tomamos en serio la incorporación de las necesidades medioambientales en la economía, eso nos obliga a cambiarlo todo en nuestra forma de producir y consumir, y en nuestra forma de trabajar y de vivir" (Klein, 2015).

\subsection{Contexto económico}

Una de las causas más señaladas respecto a esta problemática refiere que en la actualidad el modelo económico predominante es el capitalismo, el cual encuentra su base en la explotación de los recursos naturales, el abuso de la mano de obra y el consumo.

Este arquetipo de producción acelerado y automatizado es uno de los principales motivos por los que los ecosistemas se encuentran en un estado crítico a manos de la humanidad, pues no contempla al medio ambiente como una estructura vital para la supervivencia humana, sino que lo ve a manera de una mercancía más a ser apropiada y explotada. Podría decirse entonces que el capitalismo únicamente considera a la biosfera como una fuente para generar riquezas, sin considerar las consecuencias que acarrea la obtención de estas.

El calentamiento global es un fenómeno mundial pero que se manifiesta de manera muy visible en regiones específicas, generalmente de bajo desarrollo, asimismo, es un fenómeno de largo plazo, con un elevado nivel de incertidumbre. Existe un nivel de riesgo elevado y, en este sentido, se convierte desde el punto de vista económico, en un problema que trunca las posibilidades de crecimiento a futuro. Desde el aspecto monetario urgen planes de desarrollo que permitan mitigar la problemática, tanto para garantizar la subsistencia de la humanidad como la preservación de un sistema económico.

No obstante, dentro de los avances que el capitalismo ha aportado para la sustentabilidad ambiental se encuentran la generación de energías verdes o renovables, tales como la eólica o la creciente comercialización de paneles solares, la optimización de la indumentaria que requiere combustibles fósiles y la creciente industria automotriz eléctrica. Sin embargo, estos avances no logran resolver de manera satisfactoria las problemáticas ambientales, pues se enfrentan al factor de que el deterioro es una acción que se ha efectuado durante décadas y pretender remediarlo en un par de año resulta irreal.
Se habla también de otras alternativas al sistema económico actual, como la economía verde, que pretende incluir regulaciones y la utilización de instrumentos económicos para compensar el daño ambiental. Sin embargo, no se puede dejar de considerar que este tipo de economía puede operar como dispositivos tranquilizadores con la ilusión de dar respuesta a los cuestionamientos ambientales del capitalismo (Lander, E. 2011).

Asimismo, también se encuentra otra alternativa apoyada por la cultura digital: el valor social de compartir y la organización de manera horizontal, siendo esta la economía del bien común, encontrada en el Internet de las Cosas (IdC), la unión del internet de las comunicaciones, de la energía y de la logística (Rifkin, 2015). Esta genera una productividad tal que el costo por la producción de diversos servicios es cercana a cero, además de que se mantiene de manera virtual por lo que el uso de los recursos naturales disminuiría a gran escala. Esta alternativa da pie a que la metodología del comercio capitalista pueda quedar obsoleta, además de que mejoraría la productividad y se podría obtener un rendimiento sostenible de los recursos naturales.

\subsection{Contexto político}

Cuando se trata de la preservación de la biosfera, el gobierno, uno de los principales pilares de la política, deja de ser un actor visible, pues son pocos los países que tienen en su agenda realizar leyes o acuerdos que impacten de manera radical en el problema del calentamiento global. Igualmente, pese a que hay una gran cantidad de tratados internacionales en pro de la sustentabilidad y la ecología, estos no repercuten tanto como se esperaría pues su difusión es mínima y aún existen países que se resisten a unirse a la causa. Esto debido a que entre sus objetivos impera la lógica del beneficio y la ganancia por encima de la empatía hacia la biosfera.

No obstante, dentro de un país con leyes e instituciones que puedan enfocarse al medio ambiente, algunos de los grandes inconvenientes puede ser la falta de eficiencia política, la ausencia de una educación ambiental eficaz en todos los niveles escolares, así como el desinterés ciudadano, ya que de la misma manera en que el gobierno tiene la responsabilidad de interesarse por dicha problemática, la población tiene que hacer lo mismo. En un mundo globalizado y consumista, 
problemas como el calentamiento global son un hecho que ya no se puede ocultar.

\subsection{Contexto social}

El ser humano siempre se ha mirado como el centro de atención de los eventos importantes que suceden en la Tierra. Mientras los individuos iban descubriendo sus necesidades, fueron manipulando la naturaleza a su favor, para así poder hacer más fácil su desarrollo. Actualmente, los seres humanos controlan, incluso, la cantidad de animales que nacen, y cuál es el propósito de estos en la Tierra. El hombre ha perdido el respeto por la naturaleza y sus procesos, sobreexplotando al planeta, para esto se ha ido valiendo de diversas herramientas, la más actual es la tecnología. Por un lado, con esta, los procesos de explotación se han acelerado e incrementando. Por el otro, con la aparición del Internet, la sociedad actual ha encontrado la manera de expresarse, de organizarse e incluso de manifestarse contra la explotación misma de los recursos.

El internet no sólo cumple una función como herramienta para la socialización y la interacción entre personas, aunque estas no se encuentren frente a frente, sino que, también, puede servir como una plataforma para la gestación de diversas manifestaciones culturales, e incluso de movimientos sociales. Este medio no es sólo una herramienta útil que se puede utilizar simplemente porque está ahí, sino que, además, se adapta a las características básicas del tipo de movimientos sociales que están surgiendo en la era de la información (Castells, 2001).

En cuanto a la educación ambiental, el Internet, encaminado de la manera en que los movimientos sociales lo han hecho, podría revolucionar las costumbres de consumo de los seres humanos, además, de su preocupación por el planeta. Si los movimientos ecologistas actuales convergieran en un movimiento horizontal, que busque el restablecimiento de la naturaleza como un elemento vital para la vida humana, podría crearse un movimiento de carácter global, que incluya a más de una nación, e incluso a más de un continente.

\subsection{Contexto cultural}

La cultura es un factor importante que representa la conciencia y pensamiento de los seres humanos llevados a acciones cuando del tema ambiental se trata, además, permite dar un avistamiento hacia el futuro a partir de la ruta que se va dando con los hábitos que adopta la sociedad. La cultura puede permitir el desarrollo social, sin embargo, puede también tornarse en un factor negativo para la existencia futura del planeta.

En la actualidad, se puede hablar de una cultura adoptada con hábitos que excluyen por completo la importancia de la vida de otros seres, flora y fauna, quienes brindan recursos a la humanidad para sobrevivir. Es esta misma cultura del consumo la que ha dado a entender que estos recursos naturales son infinitos, sin embargo, no es así.

Ante dicha situación es importante generar un cambio sobre esta, concebir aquello que dé pie a una cultura biosférica. Por tanto, son necesarios nuevos valores que caractericen esta nueva etapa en la historia, con sociedades que perciban a la biosfera como parte de sí mismas, interiorizando esta idea para actuar conforme los efectos en el planeta. Sería entonces la conciencia que se genere lo que permita a estas, transformar el entorno y encontrarse con otros seres vivos de manera empática.

Aunado a lo anterior, se encuentra también la relevancia que tiene la cultura digital respecto a este tópico, pues esta es parte ya de la vida cotidiana, lugar donde los sujetos se apropian de las tecnologías digitales y detonan procesos simbólicos y materiales que reconfiguran los sistemas de producción, circulación y consumo de información (Castells, 2010). Por esta razón es posible darle pertinencia en cuanto a su uso con el fin de hacer conciencia sobre el cuidado de la biosfera. No obstante, la importancia de esta radicará en la manera en que sea aplicada a la vida cotidiana con el fin de concientizar y actuar en pro del medio ambiente.

\section{Hipótesis}

Con el objetivo de educar acerca del creciente fenómeno del calentamiento global y concientizar sobre los hábitos de consumo capitalistas a la generación Millennial, se requiere de una estrategia educomunicativa que involucre a los nuevos medios y fomente una conciencia crítica en pro del medio ambiente.

Es así como mediante esta estrategia se pretende condensar información verídica y oportuna que ayude a la mejor comprensión de los temas que actualmente atañen a la población en materia del cuidado ambiental. Se manejó un 
lenguaje adecuado para poder incidir y crear conciencia en el público objetivo, se plantearon reflexiones que contribuyen a la toma de acciones y decisiones sociales, individuales y colectivas, que beneficien a la biosfera. La difusión en plataformas digitales, tanto del contenido propio del colectivo Green Data como la creación de redes que ayudaron a fortalecer el trabajo hecho y reforzar su importancia actual, así como de visibilizar las problemáticas en torno a las principales industrias contaminantes con el fin de mejorar la relación entre el ser humano y la naturaleza.

Es por ello, que mediante la mencionada serie de iniciativas se busca alcanzar una difusión global que impacte en las prácticas de consumo de los Millennials, así como de un público más amplio, dada la dimensión mundial del calentamiento global para que, del mismo modo, exista una retroalimentación que mejore el contenido audiovisual y el manejo de información de colectivo y, por último, que al público al que se pretende llegar, forme parte de la mayoría de las visualizaciones de los diferentes materiales audiovisuales.

\section{Estrategia de comunicación educativa}

La educación en materia ambiental ha quedado relegada a unas pocas acciones, sin llegar a ser una prioridad. En décadas recientes, tras la notoriedad de los efectos negativos que ocasiona el calentamiento global, la enseñanza de la preservación del medio ambiente ha comenzado a tomar fuerza a través de manifestaciones que distan de la tradicional formación escolar.

La cultura ambiental y el aprendizaje de la preservación biosférica deberían ser prioridad para la sociedad, haciendo uso de herramientas multimedia, se pueden crear canales educativos para cubrir la necesidad de una educación ambiental efectiva.

Actualmente la sociedad está inmersa en lo digital, es por ello que el proyecto Green Data ha concebido una estrategia de educación informal con ayuda de las nuevas tecnologías para abordar la problemática del calentamiento global. Asimismo, Green Data se enfoca a dos principales vías de comunicación, Facebook y YouTube, dos de las redes sociales más utilizadas en este tiempo, pues ambas permiten subir y compartir contenido audiovisual.
Los Millennials, población nacida entre los años 1982 a 1996, ocupan un papel determinante dentro del acotamiento de la audiencia, pues al ser Green Data un proyecto enfocado a la producción de mensajes elaborados y distribuidos mediante la red, es necesario dirigirse a un sector capaz de desenvolverse y entenderse en dicho canal. Pese a que esta generación coexiste con generaciones previas y posteriores, en la actualidad, representan un papel clave en relación a actividad académica y profesional, pues de acuerdo al portal de noticias El País, para el año 2025, los Millennials serán más el $70 \%$ de la fuerza laboral (El País, 2017), por lo tanto, son ellos quienes se encuentran en una etapa formativa gracias a la cual, en los próximos años, tendrán la posibilidad de tomar decisiones capaces de aportar un peso considerable dentro de las acciones sociales y organizativas. Por dicha razón, resulta pertinente que estos tomen conciencia de las problemáticas que afectan a su entorno para que, de esta manera, las disposiciones que se lleguen a tomar en las siguientes décadas ayuden a consolidar un mejoramiento para la sociedad.

\section{Productos comunicativos}

Dentro de la estrategia comunicativa del proyecto Green Data, se desarrolló la opción de hacer productos comunicativos audiovisuales que pusieran en marcha esto a través de las redes, provocando una difusión que fuera más allá de lo local, lo cual implicó que dichos productos fueran realizados con diversas formas y estilos.

Para reflejar en una imagen lo que es Green Data y que la audiencia pueda tener una idea clara de lo que dicho colectivo representa, es importante que tanto los colores, como la forma y los elementos que constituyen la identidad gráfica sean lo más claros posibles y que, a su vez, representen a la naturaleza y a la tecnología, dos de los elementos centrales que dan sentido y dirección al contenido realizado dentro de este proyecto.

Al iniciar el proyecto educativo, se llevaron a cabo diversas emisiones de radio por internet, donde los usuarios tuvieron la oportunidad de escuchar el programa Green Data Radio, en donde se emitía contenido referente a tópicos ambientales, como cápsulas informativas, entrevistas a expertos en el tema, además de contar con una breve sección musical y la conducción del programa por parte de dos de los 
integrantes del equipo, quienes cada semana se encargaron de desarrollar un tema ambiental durante todo el programa.

Posteriormente, se creó una página web con la finalidad de que el colectivo contara con un registro de todos los materiales educativos que se fueran realizando. En cuanto a los contenidos audiovisuales, estos fueron previamente pensados y realizados bajo diversos parámetros que deben cumplirse para mantener un estilo que permita a los espectadores la identificación constante de los materiales audiovisuales, para así generar un grado de familiaridad que les permita reconocer y recordar los videos como un momento de aprendizaje y de sensibilización para con la biosfera, en donde se concientiza sobre diversos temas ambientales pero, principalmente, sobre el calentamiento global.

Para dicho proyecto, se tomó la decisión de abarcar tanto Facebook como YouTube, principales plataformas utilizadas por Millenials y gran parte del público en general. En cuanto a las características de los productos educomunicativos se plantearon diversos parámetros que permitieran al público reconocer el contenido del colectivo y lograr un mayor alcance, teniendo categorías como: Tutoriales, Difusión de proyectos y entrevistas y Cápsulas informativas; en cuanto a la duración de estos se permitió un rango de dos a siete minutos; respecto al idioma, los videos se realizaron en español con subtítulos en inglés; las entradas y salidas de los productos tuvieron una identidad que se repetía en cada categoría. Siendo estas las principales características, se realizaron 16 productos educomunicativos con temática medioambiental.

\section{Resultados}

Para comprender el efecto del proyecto educomunicativo: Green Data, es importante recordar que este se llevó a cabo mediante la difusión de contenidos audiovisuales a través de plataformas digitales. Dichos productos, se realizaron con la finalidad de comprobar nuestra hipótesis de trabajo y de generar aprendizaje y concientización sobre nuestro público objetivo, tal como se ha mencionado anteriormente, sin embargo, al encontrarse en internet, pudo ser posible generar un impacto sobre otro tipo de audiencia.

De un total de 17 videos producidos, se obtuvo la cantidad de 15,500 minutos reproducidos. Mientras que la cantidad de reproducciones aumenta a casi el doble, llegando a las 35,400.
Estos números son un indicativo de cómo fue el material audiovisual el mejor canal para la transmisión del mensaje hacia el público, ya que fue la manera en que mejor reaccionó la audiencia, en comparación con otro tipo de publicaciones La media de estos videos fue alrededor de 1000 minutos de reproducción por video, mientras que, en las reproducciones totales, fue un promedio de dos mil por cada uno.

Los contenidos que publica Green Data son observados en menor o mayor medida por un amplio rango de edad que parte de los 13 años a más de 65. Además, es importante destacar que la mayoría del público de Green Data en Facebook (51\% aprox.) encaja con el público objetivo que el proyecto plantea, los Millennials, quienes en la actualidad se encuentran en un rango de edad de 21 a 33 años, siendo mujeres, principalmente (60\%). Por otro lado, en la plataforma YouTube, el público del colectivo Green Data son en su amplia mayoría personas entre 25 y 34 años (98\% del total de personas alcanzada), mujeres correspondiendo al $65 \%$ y hombres al $45 \%$ lo cual refleja un acierto en cuanto al público objetivo elegido para este proyecto.

Por último, es también importante conocer el origen de las reproducciones de los videos, las cuales en su mayoría son por reproductores insertados, o links, producto de los usuarios que comparten los videos con otros usuarios. Por lo que la estrategia de montar redes sobre redes fue benéfica para la distribución de los materiales.

\section{Conclusiones}

En la parte comunicativa, el proyecto Green Data cumplió con el objetivo principal, ya que, se logró transmitir el mensaje sobre el creciente fenómeno del calentamiento global, a través de diversos contextos y acciones cotidianas que tienen un impacto directo en la crisis ambiental que se vive hoy en día. Los productos comunicativos tuvieron un impacto mayor al esperado por el colectivo, puesto que logró que el proyecto fuera visto por poco más de 30 mil personas por mes, aproximadamente, en la plataforma Facebook, que se convirtió en la principal para la difusión de los productos audiovisuales.

El público al que se llegó principalmente, como el colectivo se lo había propuesto, fue a la generación Millennial, gracias al lenguaje audiovisual que se manejó en los productos 
y a la estrategia comunicativa que se planteó desde el inicio del proyecto, donde todos los productos se difundirían a través de los nuevos medios, principalmente las redes sociales. Más del $50 \%$ de seguidores que tiene la página de Green Data en Facebook, son Millennials. Sin embargo, el otro 50\% de los seguidores que cuentan con un rango de edad entre los 13 y los 65 años, también corroboran que la estrategia y el lenguaje manejado por los productos fue de fácil acceso para cualquier tipo de público.

Otro de los objetivos que se cumplió fue que la estrategia comunicativa alcanzara una difusión global. Cerca del $18 \%$ de seguidores que tiene Green Data en Facebook no viven o son originarios de México, además de que un 10\% habla una lengua distinta al español. Gracias a la inclusión del idioma inglés en los productos comunicativos, el público de habla anglosajona ha ido incrementando a la fecha. La creación de redes, que se planteó como una parte medular dentro de la difusión del proyecto, provocó que diversos estudiantes alrededor del mundo comenzaran a interactuar con el mismo.

Aún queda un largo camino, pero los resultados han demostrado que un cambio que fomente la empatía biosférica, es posible. El interés por la preservación del medio ambiente es un tópico emergente entre la población joven por lo que un proyecto como este, que busca concientizar, permite que un futuro sustentable se vuelva una realidad.

\section{Referencias}

Castells, M. (2001). La Galaxia Internet. España: Areté.

Castells, M. (2010). The Rise of the Network Society. The Information Age: Economy, Society and Culture. Vol. I. (2nd Ed.). The Atrium, West Sussex: Wiley-Blackwell.

Greenpeace. (2017). El nuevo récord del calentamiento global corrobora la necesidad urgente de tomar medidas. Marzo 20, 2018, de Greenpeace. Sitio web: https: //goo.gl/G16ZFB

Klein, N. (2015). Esto lo cambia todo. México: Paidós.

Lander, E. (2011). La economía verde: el lobo se viste con piel de cordero. Febrero 27, 2018, de Transnational Institute. Sitio web: https://goo.gl/TUdyRS

Rifkin, J. (2015). La sociedad de costo marginal cero. El internet de las cosas, el procomún colaborativo y el eclipse del capitalismo. D.F, México: Paidós. 